\title{
Clinical outcomes of hypertensive patients with COVID-19 receiving calcium channel blockers: a systematic review and meta-analysis
}

\author{
Chia Siang Kow ${ }^{1,2} \cdot$ Dinesh Sangarran Ramachandram ${ }^{2} \cdot$ Syed Shahzad Hasan ${ }^{3,4}$
}

Received: 4 August 2021 / Revised: 27 August 2021 / Accepted: 23 September 2021 / Published online: 9 November 2021

(c) The Japanese Society of Hypertension 2021

\begin{abstract}
We aimed to perform a systematic review and meta-analysis to determine the overall effect of the preadmission/prediagnosis use of calcium channel blockers (CCBs) on the clinical outcomes in hypertensive patients with COVID-19. A systematic literature search with no language restriction was conducted in electronic databases in July 2021 to identify eligible studies. A random-effects model was used to estimate the pooled summary measure for outcomes of interest with the preadmission/ prediagnosis use of CCBs relative to the nonuse of CCBs at $95 \%$ confidence intervals (CIs). The meta-analysis revealed a significant reduction in the odds of all-cause mortality with the preadmission/prediagnosis use of CCBs relative to the nonuse of CCBs (pooled $\mathrm{OR}=0.65 ; 95 \% \mathrm{CI} 0.49-0.86$ ) and a significant reduction in the odds of severe illness with preadmission/prediagnosis use of CCBs relative to the nonuse of CCBs (pooled OR $=0.61 ; 95 \%$ CI $0.44-0.84$ ), and is associated with adequate evidence to reject the model hypothesis of 'no significant difference' at the current sample size. The potential protective effects offered by CCBs in hypertensive patients with COVID-19 merit large-scale prospective investigations.
\end{abstract}

Keywords CCB $\cdot$ antihypertensive $\cdot$ mortality $\cdot$ severity

\section{Introduction}

Investigations of the effect of the preadmission/prediagnosis use of comedications in patients with coronavirus disease 2019 (COVID-19) have been a means for researchers to establish their safety and to identify therapeutic agents that could be repurposed for the treatment of COVID-19. However, recently, it has been hypothesized that using

Supplementary information The online version contains supplementary material available at https://doi.org/10.1038/s41440021-00786-z.

Chia Siang Kow

chiasiang_93@hotmail.com

1 School of Postgraduate Studies, International Medical University, Kuala Lumpur, Malaysia

2 School of Pharmacy, Monash University Malaysia, Bandar Sunway, Selangor, Malaysia

3 School of Applied Sciences, University of Huddersfield, Huddersfield, United Kingdom

4 School of Biomedical Sciences \& Pharmacy, University of Newcastle, Callaghan, Australia calcium channel blockers (CCBs) could disrupt hypoxic pulmonary vasoconstriction and thus worsen ventilation/ perfusion mismatch, which can lead to profound hypoxemia in patients with COVID-19 [1]. Nevertheless, some researchers have also commented that the vasodilatory effects of CCBs in the pulmonary and systemic vasculature could mitigate the effects of inflammation, hypercoagulation, edema, and local vasoconstriction developed as a response to SARS-CoV-2 infection, thus facilitating oxygen delivery and survival of host cells [2]. Therefore, we aimed to perform a systematic review and meta-analysis to determine the overall effect of preadmission/prediagnosis use of CCBs on the clinical outcomes in patients with COVID-19.

\section{Methods}

This study was conducted and reported according to the recommendations outlined in the Preferred Reporting Items for Systematic Reviews and Meta-Analyses (PRISMA) guidelines. Two investigators (CSK and SSH) independently conducted a systematic literature search in multiple electronic databases, including PubMed, Google Scholar, Scopus, EMBASE, and Web of Science, in July 2021. The 
search strategy was designed to identify all publications comparing clinical outcomes between the preadmission/ prediagnosis use of CCBs and the nonuse of CCBs in patients with COVID-19 and concurrent hypertension. We applied various combinations of Boolean operators by using the following keywords for our search: [(SARS-Cov-2 OR 2019-nCOv OR COVID-19 OR coronavirus) AND (calcium channel OR calcium antagonist OR amlodipine OR dihydropyridine)]. In addition, the references from narrative reviews and other systematic reviews were cross-checked to identify additional missing publications during the initial search. Studies were eligible for inclusion in our systematic review and meta-analysis if they (1) were observational studies (of any design, for example, case-control, cohort, case series); (2) included human patients with COVID-19 and hypertension; (3) compared clinical outcomes between preadmission/prediagnosis use and nonuse of CCBs; and (4) reported adjusted association estimates. We excluded preprints and editorials, commentaries, and narrative reviews that reported no original data.

The outcomes of interest were all-cause mortality and COVID-19-associated severe illness, for example, admission to the intensive care unit, the requirement of invasive or noninvasive ventilation, mortality, and/or that defined by the authors. All relevant information from the eligible studies was extracted and recorded in a predetermined data collection table. The following information was extracted from each study: first author's surname, year of publication, the country where the study was performed, sample size (the number of patients with COVID-19 being analyzed), mean/median age of the patients, number and proportion of patients with all-cause mortality, number and proportion of patients with severe illness, adjusted effect estimates, and covariates adjusted in the study. The Newcastle-Ottawa Scale was used for critical appraisal of the quality of the included observational studies. Two investigators (CSK and $\mathrm{SSH}$ ) independently evaluated the quality of studies, and a Newcastle-Ottawa Scale score of at least 7 indicated high quality. Consensus discussions between the two investigators were carried out to resolve disagreements on the inclusion of studies, extraction of study characteristics, and quality appraisal.

A random-effects model was used to estimate the pooled odds ratio (OR) for outcomes of interest between the preadmission/prediagnosis use and nonuse of CCBs at $95 \%$ confidence intervals. We examined the heterogeneity across studies using the $I^{2}$ statistic and the $\chi^{2}$ test, where $50 \%$ and $p<0.10$, respectively, were considered an indication of the presence of heterogeneity. When heterogeneity was identified, sensitivity analysis was conducted to investigate the robustness of the results by using an alternative meta-analytic model, namely, the inverse variance heterogeneity (IVhet) model. All analyses were performed using
Meta XL, version 5.3 (EpiGear International, Queensland, Australia).

\section{Results}

Our literature search yielded 547 abstracts. After deduplication and application of the eligibility criteria, 39 relevant articles were shortlisted for inclusion through full-text examination. Of these, 30 studies were excluded for reporting no adjusted association estimates, reporting no clinical outcomes, including mixed hypertensive and nonhypertensive patients without subgroup analysis on hypertensive patients, or comparing clinical outcomes between COVID-19 and non-COVID-19 patients. Eventually, nine studies [1, 3-10] were included in this systematic review and meta-analysis; eight studies [3-10] reported adjusted association estimates for all-cause mortality, while five studies [1, 4, 6, 7, 9] reported adjusted association estimates for COVID-19-associated severe illness. The study characteristics are depicted in Table 1. Meta-analysis of seven studies $[3-7,9,10]$ with a total of 8413 patients with COVID-19 revealed a significant reduction in the odds of all-cause mortality with the preadmission/prediagnosis use of CCBs relative to the nonuse of CCBs; the estimated effect indicates mortality reduction (Fig. 1; pooled OR $=$ $0.65 ; 95 \%$ confidence interval $0.49-0.86$ ) and is associated with adequate evidence to reject the model hypothesis of 'no significant difference' at the current sample size. However, sensitivity analysis with IVhet model (performed due to presence of heterogeneity) revealed no significant mortality reduction with the preadmission/prediagnosis use of CCBs relative to the nonuse of CCBs (pooled OR $=0.75$; 95\% confidence interval 0.52-1.09). Visual inspection of the funnel plot (Supplementary Fig. S1) revealed some degree of publication bias, as we found asymmetry in the scatter of studies (with more studies with positive findings than studies with negative findings), but the triangular 95\% confidence region included almost all studies, suggesting that only a small bias was present.

The definition of severe illness varied across studies; in the studies by Choksi et al. [4], Christiansen et al. [6], and Peng et al. [9], it was defined as admission to the intensive care unit; in the studies by Yan et al. [7], it was defined according to the Diagnosis and Treatment Protocol for Novel Coronavirus Pneumonia by the Chinese National Health Commission. In the study by Mendez et al. [1], it was defined as the use of invasive or noninvasive mechanical ventilation or death. Meta-analysis of four studies [4, 6, 7, 9] with a total of 2618 patients with COVID19 revealed a significant reduction in the odds of severe illness with the preadmission/prediagnosis use of CCBs relative to the nonuse of $\mathrm{CCBs}$; the estimated effect 


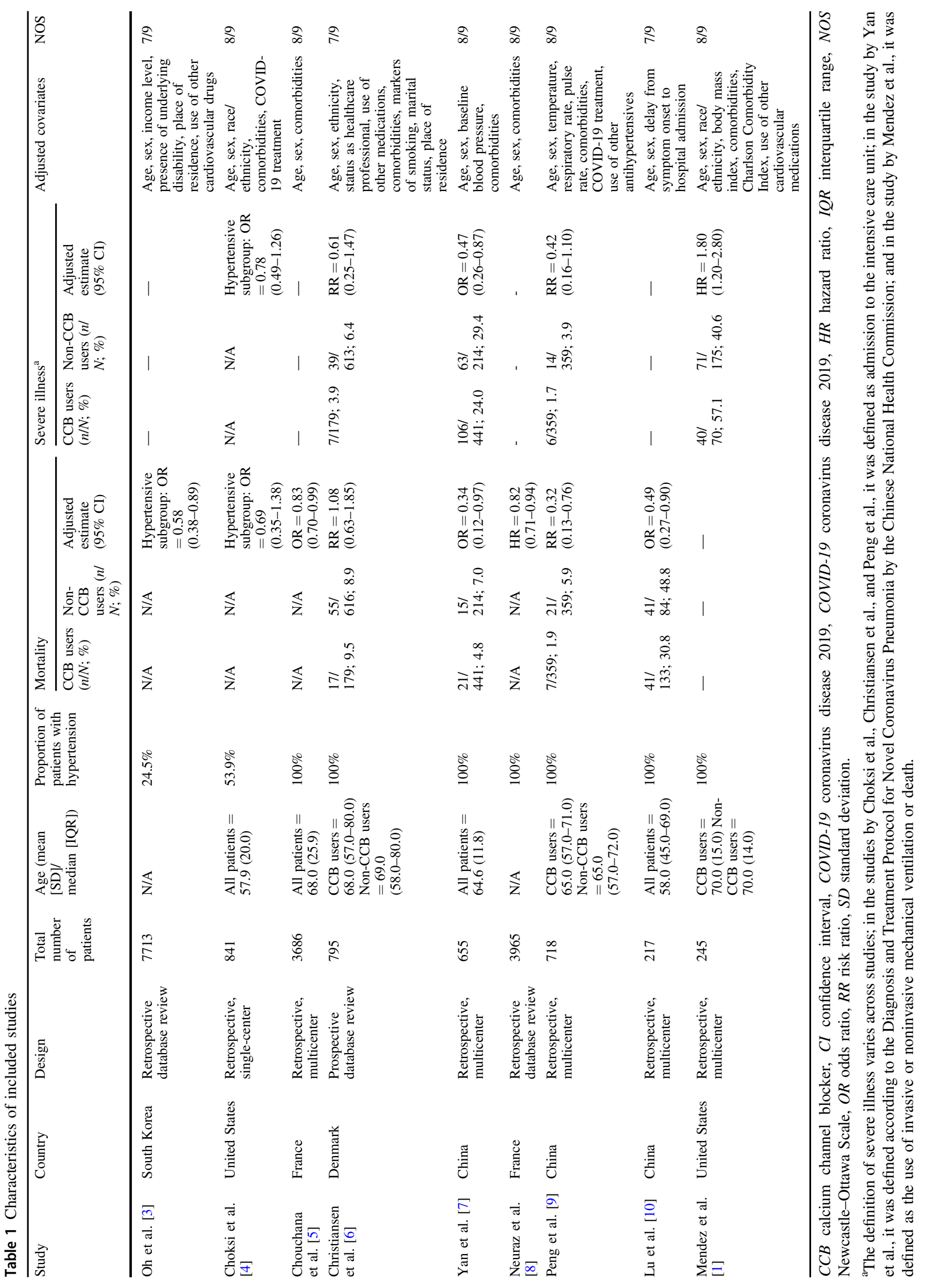



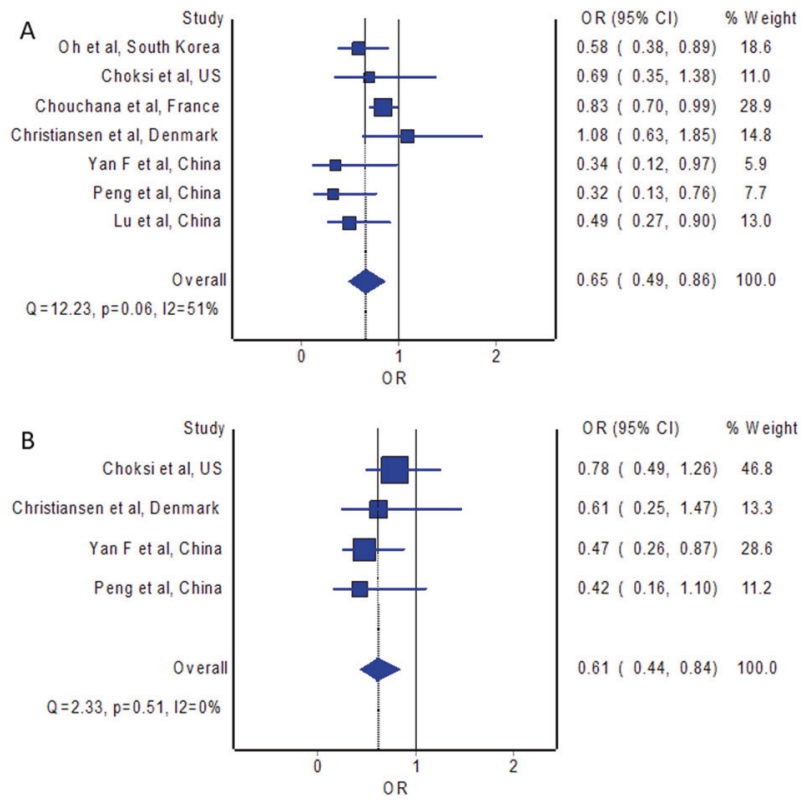

Fig. 1 Pooled odds ratio (OR) of mortality (A) and severe illness (B) between CCB users and non-CCB users with COVID-19 and concurrent hypertension

indicates a reduction in severe illness (Fig. 1; pooled OR $=$ $0.61 ; 95 \%$ confidence interval $0.44-0.84$ ) and is associated with adequate evidence to reject the model hypothesis of 'no significant difference' at the current sample size.

\section{Discussion}

Overall, the real-world studies observed a significant protective effect with the preadmission/prediagnosis use of CCBs against all-cause mortality and COVID-19-associated severe illness in patients with COVID-19 and concurrent hypertension relative to the nonuse of CCBs. Although the studies [3-10] included in our meta-analysis were of retrospective design, the potential protective effects offered by CCBs in hypertensive patients with COVID-19 merit largescale prospective investigations. Indeed, patients with hypertension are at high risk of a worse prognosis when they acquire COVID-19; the establishment of protective effects with CCBs could lead to the preferential prescription of these widely available agents for patients with a diagnosis of hypertension during the COVID-19 pandemic, and potential deaths due to COVID-19 could be averted.

It should be noted that the studies included in our metaanalysis were mostly retrospective in design, and thus, the generalizability of the findings may be limited. Further- more, our analysis focused on the preadmission/prediagnosis use of CCBs; the effect of the de novo introduction of CCBs in patients with COVID-19 cannot be ascertained. In addition to these limitations, our systematic review and meta-analysis have some strengths that should be acknowledged, including the number of studies and patients included and the performance of sensitivity analysis.

\section{Compliance with ethical standards}

Conflict of interest The authors declare no competing interests.

Publisher's note Springer Nature remains neutral with regard to jurisdictional claims in published maps and institutional affiliations.

\section{References}

1. Mendez SR, Frank RC, Stevenson EK, Chung M, Silverman MG. Dihydropyridine calcium channel blockers and the risk of severe COVID-19. Chest 2021;160:89-93.

2. Crespi B, Alcock J. Conflicts over calcium and the treatment of COVID-19. Evol Med Public Health. 2020;9:149-56.

3. Oh TK, Cho HW, Suh JW, Song IA. Incidence and mortality associated with cardiovascular medication among hypertensive COVID-19 patients in South Korea. Yonsei Med J. 2021;62:577-83.

4. Choksi TT, Zhang H, Chen T, Malhotra N. Outcomes of hospitalized COVID-19 patients receiving renin angiotensin system blockers and calcium channel blockers. Am J Nephrol. 2021;52:250-60.

5. Chouchana L, Beeker N, Garcelon N, Rance B, Paris N, Salamanca $\mathrm{E}$, et al. Association of antihypertensive agents with the risk of in-hospital death in patients with Covid-19 [published online ahead of print, 2021 Feb 17] [published correction appears in Cardiovasc Drugs Ther. 2021 Mar 4]. Cardiovasc Drugs Ther. 2021;1-6.

6. Christiansen CF, Pottegård A, Heide-Jørgensen U, Bodilsen J, Søgaard OS, Maeng M, et al. SARS-CoV-2 infection and adverse outcomes in users of ACE inhibitors and angiotensin-receptor blockers: a nationwide case-control and cohort analysis. Thorax 2021;76:370-9.

7. Yan F, Huang F, Xu J, Yang P, Qin Y, Lv J, et al. Antihypertensive drugs are associated with reduced fatal outcomes and improved clinical characteristics in elderly COVID-19 patients. Cell Discov. 2020;6:77.

8. Neuraz A, Lerner I, Digan W, Paris N, Tsopra R, Rogier A, et al. Natural language processing for rapid response to emergent diseases: case study of calcium channel blockers and hypertension in the COVID-19 pandemic. J Med Internet Res. 2020;22:e20773.

9. Peng C, Wang H, Guo YF, Qi GY, Zhang CX, Chen T, et al. Calcium channel blockers improve prognosis of patients with coronavirus disease 2019 and hypertension. Chin Med J. 2021;134:1602-9.

10. Lu QB, Jiang WL, Zhang X, Li HJ, Zhang XA, Zeng HL, et al. Comorbidities for fatal outcome among the COVID-19 patients: a hospital-based case-control study. J Infect. 2021;82:159-98. 